\title{
Measurement of Ocean Wave Heights Using the Geos 3 Altimeter
}

\author{
Clifford L. RufEnach \\ NOAA/ERL Wave Propagation Laboratory, Boulder, Colorado 80302
}

Werner R. AlPers

Institute of Geophysics, University of Hamburg and Max-Planck-Institute of Meteorology, Hamburg. West Germany

\begin{abstract}
Radar altimeter signals transmitted from the low-orbiting satellite Geos 3 were analyzed for two selected orbits over high seas associated with hurricane 'Caroline' in the Gulf of Mexico and a North Atlantic storm. The measured values of significant wave height are in reasonable agreement with surface measurements, provided that the altimeter data are properly edited. The internal consistency of estimated wave heights for the North Atlantic storm, a standard deviation of $0.6 \mathrm{~m}$ or less, and the good agreement with surface truth lend credence to the method. A statistical analysis of the pulse slope variation gives estimated values of significant wave height within $\pm 1 \mathrm{~m}$ of the true values $75 \%$ of the time for spatial averaging over $70 \mathrm{~km}$.
\end{abstract}

\section{INTRODUCTION}

Short-pulse radar altimeters aboard low-orbiting satellites have the capability of measuring the mean height of the ocean surface, the geoid, and the roughness of this surface, the wave height. The mean height measurement uses the round trip travel time of radar pulses to estimate the distance from the satellite to the surface. The roughness of the ocean surface causes stretching of the return pulse leading edge which is a direct measure of the ocean wave height (see, for example, Barrick [1972]; Brown [1977]). In the present work the significant wave height $H_{1 / 3}$ is inferred from the shape of the average received pulse.

Short-pulse altimeters are aboard two satellites, Skylab S193 and Geos 3 [McGoogan, 1975]. Both altimeters have similar pulse widths, about $15 \mathrm{~ns}$; allowing the measurement of higher wave heights, whereas the altimeter scheduled for launch aboard Seasat-A in May 1978 will have a nominal pulse width of $3 \mathrm{~ns}$, allowing measurement of lower wave heights.

Contributions to the received radar signal occur when the scattered energy from specular points on the rough ocean surface is returned to the radar antenna. However, these points will be received at different delays, since they come from different depths into the rough surface. Therefore the delayed signals add randomly, causing stretching of the leading edge of the return pulse. This scattering process for a short-pulse satellite altimeter requires that the spherical nature of the transmitted wave be considered [Moore and Williams, 1957; Barrick, 1972]. Thus the received pulse increases in amplitude after being reflected from the surface up to a peak (plateau) value. This plateau value is reached when the trailing edge of the transmitted pulse first intersects the mean surface, for a flat sea an area about $4 \mathrm{~km}$ in diameter for Geos 3, whereas for high seas it occurs when the trailing edge reaches the lowest trough, which could be 6-7 km in diameter.

The purpose of the present work is to develop a method for analyzing the Geos 3 altimeter signals in terms of ocean wave heights. The technique must take into account the peculiarities associated with the Geos 3 altimeter. Indeed, several altimeter parameters are observed in order to determine when and if the altimeter data required to calculate $H_{1 / 3}$ are reliable. A more accurate method for analyzing Geos 3 data may evolve in the future; however, it is believed that the method presented here

This paper is not subject to U.S. copyright. Published in 1978 by the American Geophysical Union.

Paper number $8 \mathrm{C} 0452$ leads to useful results. Data from two orbits were selected for analysis: overflights of hurricane Caroline in the Gulf of Mexico and a North Atlantic storm.

\section{THEORY}

The time evolution of the backscattered signal as recorded by the receiver is given by the convolution of the incoming pulse $I_{0}(t)$ with the impulse response function $H(t)$ :

$$
I(t)=H(t) * I_{0}(t)=\int_{-\infty}^{+\infty} H\left(t-t^{\prime}\right) I_{0}\left(t^{\prime}\right) d t^{\prime}
$$

where $H(t)$ is the convolution of the radar sea surface impulse response function $S(t)$, which describes the backscattering of the pulse and the receiver response function $R(t)$ :

$$
H(t)=S(t) * R(t)
$$

The antenna beam width effects were not included, since they have a negligible effect on the shape of the leading edge of the pulse.

For the simplified case, a nearly smooth flat sea surface, the mean backscattered signal is proportional to the area illuminated by the pulse. For this simplification the signal energy is a linear function of time if $t_{0}-\tau / 2<t<t_{0}+\tau / 2$ and remains constant for $t>t_{0}+\tau / 2$ [Brown, 1977]. Assuming that the surface impulse response $S(t)$ is a step function, the receiver response $R(t)$ is a delta function, and the incoming pulse is rectangular, then a linear response is obtained as shown by the solid line in Figure 1.

If the sea surface is rough, however, specular points are encountered above and below mean sea level. This causes the linear rise to be stretched as illustrated by the dashed line in Figure 1. For a rough sea the surface response function $S(t)$ is then the convolution of a step function and the probability density function $\boldsymbol{P}_{\mathbf{3}}(\zeta, n)_{n=0}$ for specular points having heights $\zeta$ above the mean level (n represents the vector normal of the specular points). Specular points for a nadir-looking altimeter are characterized by tangent planes on the ocean waves where the normals of these planes are vertical to the mean sea surface; i.e., $\mathbf{n}=\left(\eta_{x}, \eta_{y}\right)=0$ [Barrick, 1968].

In the analysis of a rough surface we can assume that the ocean wave field obeys Gaussian statistics and that the backscattered energy per unit illuminated area is independent of height above the mean sea level. Shapiro et al. [1972] have 


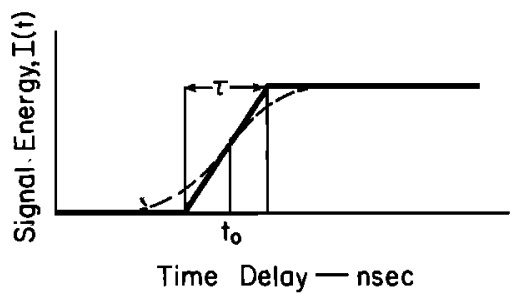

Fig. 1. Simplified representation of average received pulse shape.

shown evidence that the radar energy near vertical incidence increases linearly with increasing distance below the wave crests. However, these small effects are unlikely to be detectable with the Geos 3 altimeter because of its limited resolution.

The ocean wave field is assumed to be Gaussian distributed with independent wave heights and the wave normals,

$$
P_{\mathrm{s}}(\zeta, n)=P_{1}(\zeta) P_{2}(n)
$$

For a nadir-looking altimeter, (2) can then be represented by [Brown, 1977]

$$
P_{8}(\zeta, n)_{n=0} \sim \exp -\zeta^{2} / 2 \sigma_{\theta}{ }^{2}
$$

where $\sigma_{s}$ is the rms wave height and is related to $H_{1 / 3}$ by

$$
\sigma_{s}=1 H_{1 / 3}
$$

In this analysis it is implied that the reflected pulse contains contributions from so many specular points that the statistics of the returned pulse envelope can be described by a Rayleigh distribution or, equivalently, its square by an exponential distribution.

The receiver response function is useful in characterizing the effect 'of pulse jitter on the average received pulse shape. The altimeter pulse acquisition requires continuous adjustment of the individual pulse positions. The random portion of this adjustment is termed pulse jitter and may be assumed to be Gaussian distributed with an rms value $\sigma$, [Hofmeister et al., 1976]. Therefore the receiver response function $R(t)$ is approximately

$$
\dot{R}(t) \sim \exp \left[-\left(t-t_{0}\right)^{2} / 2 \sigma_{j}{ }^{2}\right]
$$

\begin{tabular}{|c|c|}
\hline Characteristic & Value \\
\hline \multicolumn{2}{|l|}{ Orbital } \\
\hline $\begin{array}{l}\text { Mean altitude } \\
\text { Inclination } \\
\text { Eccentricity } \\
\text { Period } \\
\text { Satellite velocity }\end{array}$ & $\begin{array}{l}843 \mathrm{~km} \\
115^{\circ} \\
0 \\
101.8 \mathrm{~min} \\
\sim 7 \mathrm{~km} / \mathrm{s}\end{array}$ \\
\hline \multicolumn{2}{|l|}{ Radar Altimeter } \\
\hline $\begin{array}{l}\text { Transmitting frequency } \\
\text { Pulse width (one-half power width)* } \\
\text { Pulse width }\left(\sigma_{\tau}=e^{-1 / 2} \text { width) } \dagger\right. \\
\text { Pulse repetition frequency } \\
\text { Detector characteristic } \\
\text { Antenna beam width (one-half power) } \\
\text { Tracking loop jitter }\left(\sigma_{J}\right)^{*}\end{array}$ & $\begin{array}{l}13.9 \mathrm{GHz} \\
14-15 \mathrm{~ns} \\
6.2 \mathrm{~ns} \\
100 \text { pulse/s } \\
\text { square law } \\
2.6^{\circ} \\
\sim 4 \mathrm{~ns} \text { for } H_{1 / \mathrm{s}} \simeq 2 \mathrm{~m} \\
\sim 9 \mathrm{~ns} \text { for } H_{1 / \mathrm{s}} \cong 10 \mathrm{~m}\end{array}$ \\
\hline
\end{tabular}

and the incoming pulse can also be approximated by a Gaussian shape with rise time $\sigma_{r}$ :

TABLE 1. Geos 3 Satellite Characteristics

*Based on electrical tests of the flight model altimeter [Hofmeister et al., 1976].

†Based on one-half power width of $14.6 \mathrm{~ns}$.

$$
I_{0}(t) \sim \exp \left(-t^{2} / 2 \sigma_{\tau}^{2}\right)
$$

The average return pulse includes the convolution of three Gaussian functions:

$$
P_{3}(\zeta=c t / 2, n)_{\eta=0} \quad R(t) \quad I_{0}(t)
$$

where $c$ is the speed of light. The resulting Gaussian expression is $G_{\sigma_{\mathrm{c}}}(t)$ with rise time

$$
\sigma_{c}=\left[\left(2 \sigma_{s} / c\right)^{2}+\sigma_{\tau}^{2}+\sigma_{J}^{2}\right]^{1 / 2}
$$

Therefore the resulting analytical expression for the average return signal is

$$
\begin{aligned}
I(t)=\int_{-\infty}^{\infty} \theta\left(t-t_{0}-t^{\prime}\right) G_{\sigma_{\mathrm{c}}}\left(t^{\prime}\right) d t^{\prime} \\
\quad=\frac{I_{\infty}-I_{-\infty}}{2}\left(1+\operatorname{erf} \frac{t-t_{0}}{(2)^{1 / 2} \sigma_{c}}\right)+I_{-\infty}
\end{aligned}
$$

where

$$
\begin{array}{cc}
\theta\left(t-t_{0}\right)=I_{\infty} & t>t_{0} \\
\theta\left(t-t_{0}\right)=I_{-\infty} & t<t_{0}
\end{array}
$$

and erf $t=2 /(\pi)^{1 / 2} \int_{0}^{t} e^{-\lambda z} d \lambda$ is the error function.

From (4) and (8) we obtain

$$
H_{1 / 8}=0.6\left(\sigma_{c}{ }^{2}-\sigma_{\tau}{ }^{2}-\sigma_{f}^{2}\right)^{1 / 2}
$$

where $H_{1 / s}$ is in meters and $\sigma_{c}, \sigma_{\tau}$, and $\sigma_{j}$ are in nanoseconds. Equations (9) and (10) are used to deduce the significant wave height in the examples examined.

\section{DESCRIPTION OF AltiMETER}

The Geos 3 satellite was launched on April 9, 1975, into a circular orbit with each consecutive orbit precessing about $25^{\circ}$ to the west. The principal orbital and altimeter characteristics are given in Table 1 . The tabulated altimeter characteristics refer to the instantaneous short-pulse (intensive) mode of operation relevant for sea state studies. The altimeter probes the ocean surface 100 times per second resulting in a spatial period of $70 \mathrm{~m}$. Each radar pulse is sampled in the radar receiver by 16 sampling gates as illustrated in Figure 2. These gates of a width of $12.5 \mathrm{~ns}$ and uniformly spaced at $6.25 \mathrm{~ns}$ intervals sample the pulse at discrete levels of $8 \mathrm{mV}$. Furthermore, the receiver continuously adjusts the position of these 16 gates to track the leading edge of the return pulse, which gives a measure of the satellite altitude. This tracking adjustment is accomplished by a split-gate tracking loop which minimizes the loop error voltage $E=2 I_{10}-I_{\infty}$, where $I_{10}$ is the sampled energy in gate number 10 and $I_{\infty}$ is the sampled energy in the plateau region (see Figure 2). The loop bandwidth is approximately $4 \mathrm{~Hz}$, which means that any real or apparent changés in

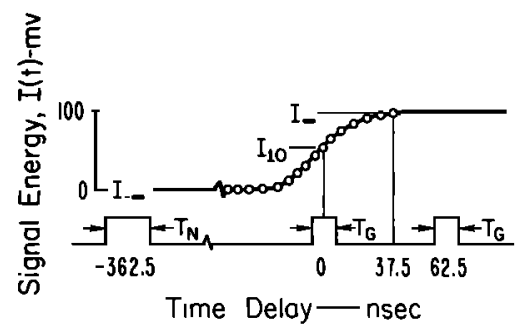

Fig. 2. Geos 3 sampling gate configuration for intensive mode: $T_{G}$ $=12.5 \mathrm{~ns}, T_{N}=200 \mathrm{~ns}$. $I_{10}$ is the sampled energy gate $10, I_{\infty}$ is the sampled energy plateau region, and $I_{-\infty}$ is the sampled energy noise region. 
altitude slower than $4 \mathrm{~Hz}$ will appear as a tracking adjustment.

The random fluctuations in this error voltage, termed jitter, are important for sea state studies, since jitter in position of the individual pulses causes additional stretching of the average received pulse. The expected standard deviation of tracking loop jitter is about 4 ns for low sea states and increases to about $9 \mathrm{~ns}$ for high sea states (see Table 1).

The 16 sampling gates are also available through a resistance-capacitance low-pass filter with a 1-s time constant. These average gate values, hereafter designated as analog averages, are sampled at discrete levels of $2 \mathrm{mV}$ and transmitted to the earth every $3.2 \mathrm{~s}$.

Several other parameters are used to determine if the altimeter was operating properly or might bias the estimates of $H_{1 / 3}$. These parameters include (1) altimeter status, (2) satellite altitude, (3) position of the average received pulse relative to the 16 sampling gates $t_{0}$, and (4) Automatic Gain Control (AGC), which is a measure of received signal level.

\section{ANALysis Method}

The analysis method is separated into (1) the statistical analysis of data and (2) the development of criteria for deleting unreliable data that could bias the estimates of $H_{1 / 8}$.

The average pulse shapes were obtained by digitally averaging 320 instantaneous pulses over $3.2 \mathrm{~s}$, defined as one frame of data. This corresponds to a spatial resolution of about $23 \mathrm{~km}$, the distance the satellite travels in $3.2 \mathrm{~s}$. Other statistical parameters, i.e., variance, skewness, kurtosis, intergate, and interpulse correlation coefficients, were calculated, and the results are given in Appendix A.

The average received pulse shape is represented by the error function of (9),

$$
I\left(t_{t}\right)=\frac{I_{\infty}-I_{-\infty}}{2}\left(1+\operatorname{erf} \frac{t-t_{0}}{(2)^{1 / 2} \sigma_{c}}\right)+I_{-\infty}
$$

where the parameters $I_{\infty}$ and $I_{-\infty}$ are available from the altimeter output, average plateau, and noise gate energies, respectively; $t_{i}$ is the time delay of the transmitted pulse as shown in Figure 3, and $\sigma_{c}$ is a measure of the pulse stretching. The pulse stretching is given in Figure 3 for $0 \leq H_{1 / 3} \leq 12 \mathrm{~m}$ based on (10) and (11).

The significant wave height $\left(H_{1 / 9}\right)$ is estimated from the average pulse shape by a standard least square error analysis using gate 10 and the four adjacent gates. This analysis was performed for the analog and digitally averaged gates. The locations of the five points selected for the analysis are shown with open circles in Figure 3.

The form of the least square error is

$$
\epsilon=\sum_{l=8}^{12}\left[I\left(t_{l}\right)-I_{l}\right]^{2}
$$

where $I_{i}$ represents the average gate values. The minimization was numerically performed by selecting successive values of $\sigma_{c}$ and $t_{0}$.

The justification for selecting only five points is as follows: The results of intergate correlation analysis for several different orbits show an unexpected and consistent decrease in correlation between gates 13 and 14 and a corresponding unexpected increase in correlation between gates 12 and 13 as illustrated in Figure A1. These correlation coefficients show that the position of gate 13 is mislocated with a delay nearly identical to that of gate 12 . Therefore including gate 13 in the analysis could bias the estimates.

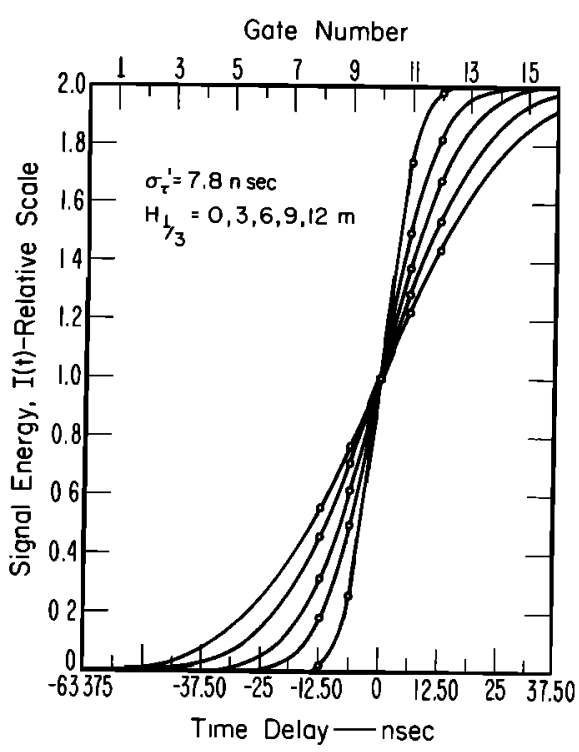

Fig. 3. Theoretical shape of received pulse for Geos 3 altimeter. Open circles indicate the four sampled points (gates) adjacent to gate $10, \sigma_{\tau}{ }^{\prime}=\left(\sigma_{\tau}^{2}+\sigma_{j}{ }^{2}\right)^{1 / 2}, I_{\infty}=2, I_{-\infty}=0$, and $t_{0}=0$.

The jitter is calculated from the range servo error voltage $y_{i}$. The servo error voltage is proportional to the satellite altitude rate (E. L. Hofmeister, private communication, 1977). Therefore the random jitter fluctuations are obtained first by calculating the running sum, $z_{i}=\sum_{j=1}^{l} y_{j}$, where $y$ is given in centimeters. The resulting values are then detrended and converted to time units by using $t_{j}(\mathrm{~ns})=y_{\lambda}(\mathrm{cm}) / 15$ to obtain the jitter fluctuations. In the present work a quadratic least square error analysis was used to subtract both altitude rate and acceleration. Similar results were obtained by using several slightly different analytic methods.

The Geos 3 overflight (orbit 2024) of hurricane Caroline was selected to illustrate the method of analysis. This includes the least square analysis described above to estimate $H_{1 / 8}, t_{0}$, and $\epsilon$. In addition, the altimeter status and the satellite altitude were monitored in an attempt to determine when the altimeter data should be edited. Figure 4 shows the result of this analysis. Figure $4 a$ shows the variation of $\epsilon$, the mean square error which is a measure of the goodness of fit to the error function. Figure $4 b$ shows the variation of $t_{0}$, which is a measure of how effectively the altimeter receiver is positioning the incoming pulses. Figure $4 c$ shows the standard deviation of the jitter $\sigma_{j}$, which is a measure of the random time shifts between instantaneous pulses. This jitter is expected to increase with increasing $H_{1 / 3}$ and decreasing signal level. Figure $4 d$ shows the variations of the satellite altitude with four values of altitude averaged to obtain altitude values every $0.4 \mathrm{~s}$. Finally, and most importantly, Figure $4 e$ gives the estimates of $H_{1 / \mathrm{s}}$. The solid circles represent the $H_{1 / \mathrm{s}}$ values based on an effective pulse width $\sigma_{\tau}{ }^{\prime}$ $=\left(\sigma_{\tau}^{2}+\sigma_{j}^{2}\right)^{1 / 2}=7.8 \mathrm{~ns}$, whereas the open circles represent $H_{1 / 3}$ values based on measured values of $\sigma_{j}$ from the method described above using (10). Both methods of calculating $H_{1 / \mathrm{s}}$ use (10) with $\sigma_{\tau}=6.2 \mathrm{~ns}$. The dashed lines refer to portions along the ground track where the data were deleted by using the editing criteria described below.

The principal features of Figure 4 are the large negative spikes in satellite altitude (see points A, B, and C); these spikes are directly correlated with large values of $\sigma_{\jmath}$. The estimates of $H_{1 / 3}$ without jitter correction exhibit less deviation from adjacent values than estimates with jitter correction exhibit for the 


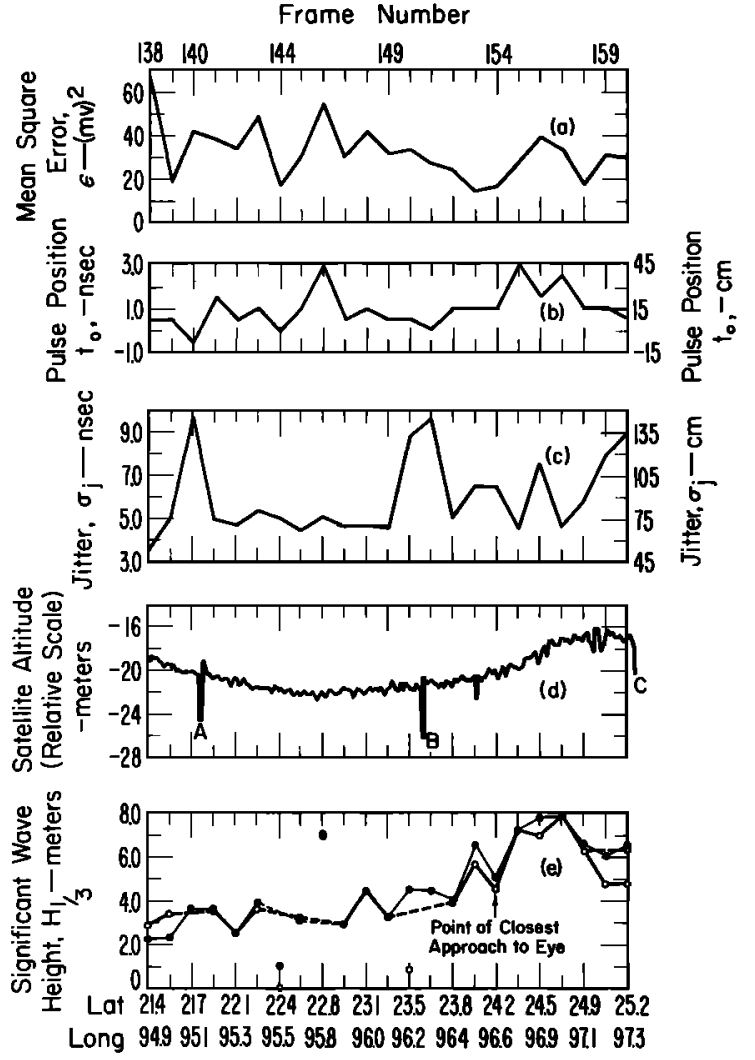

Fig. 4. Plots of altimeter parameters from selected portions of orbit 2024 in the Gulf of Mexico near hurricane Caroline, August 31, 1975. Solid circles represent $H_{1 / 3}$ based on $\sigma_{\tau}{ }^{\prime}=7.8 \mathrm{~ns}$. Open circles

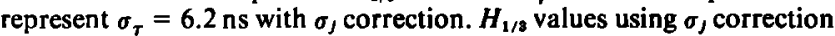
underestimate the wave height when negative spikes are present in satellite altitude (e.g., $\sigma_{\tau}{ }^{\prime}>\sigma_{c}$ for frames 140 and 150). Points A, B, and $C$ indicate negative spikes in altitude.

negative spike associated data The smaller values of $t_{0}$ appear to correlate with these negative spikes.

A similar analysis was undertaken for orbit 4546 with data over a much larger area and range of sea states. The results from this North Atlantic orbit are shown in Figure 5. First, the satellite altitude output did not exhibit the large negative spikes present for orbit 2024. Therefore continuous unbiased estimates of $H_{1 / 3}$ are available except for a small area near frame 15 where the signal level dropped below the minimum threshold. The parameters selected for display in Figure 5 are $t_{0}, \sigma_{l}$, and $H_{1 / 3}$. The values of $H_{1 / 3}$ were computed by using a three-frame running average resulting in a spatial resolution of about $70 \mathrm{~km}$; the other parameters are based on one-frame averages or $23-\mathrm{km}$ resolution. The pulse position $t_{0}$ appears to decrease with decreasing sea state from approximately $t_{0} \cong 2$ $\mathrm{ns}$ to $1 \mathrm{~ns}$ as is illustrated in Figure $5 a$. Figure $5 b$ illustrates typical values for jitter $\sigma$, of about $8 \mathrm{~ns}$ for high sea states. These values gradually decrease with decreasing sea states to 4 or $5 \mathrm{~ns}$. These results are in agreement with tests on the flight model altimeter. The estimates of $H_{1 / \mathrm{s}}$ using analog-averaged pulse shapes exhibit wider scatter than digital averages with or without jitter correction. Therefore only the digital-averaged values are used here.

The following criteria were applied to edit data that might bias the $H_{1 / 8}$ estimates. First, and most important, data associated with large negative spikes in satellite altitude were edited when the peak values of the spikes were greater than $2 \mathrm{~m}$, e.g., see frames 140, 150, 151, and 160 of orbit 2024.
Two other parameters that have indicated bias are $t_{0}$ and a nonmonotonic shape for the average pulse in the plateau region. The values of $t_{0}$ that bias $H_{1 / 3}$ are taken as $t_{0}<1 \mathrm{~ns}$ or $t_{0}$ $>3 \mathrm{~ns}$ as described in Appendix B. Deleting data based on pulse shape may seem somewhat arbitrary; nevertheless, useful results were obtained after carefully analyzing the data, leading to the criterion to delete data when $\left|I_{\max }-I_{\min }\right|>10 \mathrm{mV}$ in the plateau region where the average values of $I_{\max }$ are usually located near gates 12 or 13 and $I_{\min }$ near gates 15 or 16 . The values of $I_{\max }$ and $I_{\min }$ were calculated every $3.2 \mathrm{~s}$.

The criteria $\left|I_{\max }-I_{\min }\right|>10 \mathrm{mV}$ and $t_{0}<1 \mathrm{~ns}$ or $t_{0}>3 \mathrm{~ns}$ may delete valid data occasionally. For example, the standard deviation of $I_{\max }$ or $I_{\min }$ is $1 /(320)^{1 / 2}=5.6 \%$ of the mean value which for typical plateau values of $90 \mathrm{mV}$ implies a standard deviation of about $5 \mathrm{mV}$. Therefore if one gate is greater than one standard deviation high and another is less than one standard deviation low, valid data would be deleted.

\section{RESULTS}

Two orbits were selected for estimating $H_{1 / 3}$ during high sea conditions. The subsatellite tracks for these orbits are shown in Figure 6. Figure 6a illustrates the track for orbit 2024 at 0100 UT on August 31, 1975, near hurricane Caroline. The point of closest approach to the eye (point A) is about $40 \mathrm{~km}$. Figure $6 b$ illustrates the track for orbit 4546 when it passed near a low-pressure system associated with a North Atlantic storm at 0800 UT on February 25, 1976.

The variation in $\boldsymbol{H}_{1 / 3}$ along the ground track for hurricane Caroline shows the following general variations provided the data are properly edited (see Figure $4 c$ ). The values are nearly constant for distances of 300 to $100 \mathrm{~km}$ (frames 140-150) from the eye; beyond this point, however, there is a gradual trend for $H_{1 / 3}$ to increase up to a maximum (frame 156) about $40 \mathrm{~km}$ beyond the point of closest approach. The motion of the hurricane toward the north and west is consistent with an asymmetry in $H_{1 / 3}$ around the point of closest approach based on wind-driven waves, since the counterclockwise circulation causes larger winds in the northeast quadrant (hurricane winds are known to increase up to a maximum corresponding to a radius typically $25 \mathrm{~km}$ ). For orbit 2024 the ground track passes close to the radius of maximum winds at the point of closest approach. These results are in agreement with expected values based on wave model analysis of similar hurricanes [Cardone

Frome Number

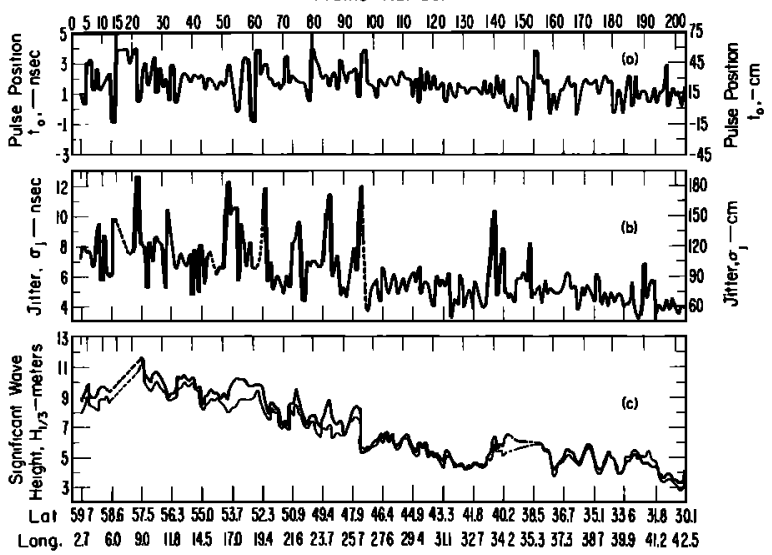

Fig. 5. Plots of altimeter parameters from orbit 4546 in the North Atlantic, February 25,1976 . Heavy line represents $H_{1 / 8}$ based on $\sigma_{\tau}{ }^{\prime}=$ $7.8 \mathrm{~ns}$. Light line represents $H_{1 / \mathrm{a}}$ based on $\sigma_{\tau}=6.2 \mathrm{~ns}$ with $\sigma_{j}$ correction. Here $\sigma_{\tau}{ }^{\prime}=\left(\sigma_{\tau}^{2}+\sigma_{\rho}^{2}\right)^{1 / 2}$ 
et al., 1976]. No ship reports were available near the satellite track.

The estimates of $H_{1 / \mathrm{s}}$ for orbit 4546 are shown in Figure 7, where less data editing was required. Therefore a continuous plot is available, illustrating a definite trend: At the beginning of the orbit the wave heights are near $9 \mathrm{~m}$, increasing to about $10 \mathrm{~m}$ before gradually decreasing to about $4 \mathrm{~m}$. The scatter in the values is considered to be internally consistent and reasonable. The standard deviation is calculated to be about $0.6 \mathrm{~m}$ or less over the entire ground track. It was based on a quasi-linear regression analysis, the data being separated into three linear segments.

Ship reports and surface weather maps (available from NOAA/EDS, National Climate Center, Asheville, North Carolina, 28801) are compared with orbit 4546 . The (0600 UT) northern hemisphere surface chart was analyzed for February 25 , as well as the charts from the 2 previous days, along the subsatellite path. Both the isobar contours and the ship reports were used to estimate the magnitude of the wind. The winds near the northern coast of Scotland had been blowing for 50 hours with velocities of about $20 \mathrm{~m} / \mathrm{s}$. Therefore the waves were considered to be fully developed or nearly fully developed; see, for example, Kinsman [1965]. Wave heights for a fully developed sea correspond to a nondimensional peak frequency $\nu=0.13$ as given by Hasselmann et al. [1976] and adjusted by Pierson [1977]:

$$
H_{1 / 9}(\mathrm{~m})=9.4 \times 10^{-4} \nu^{-10 / 8} U_{10}^{2} \mathrm{~m} / \mathrm{s}
$$
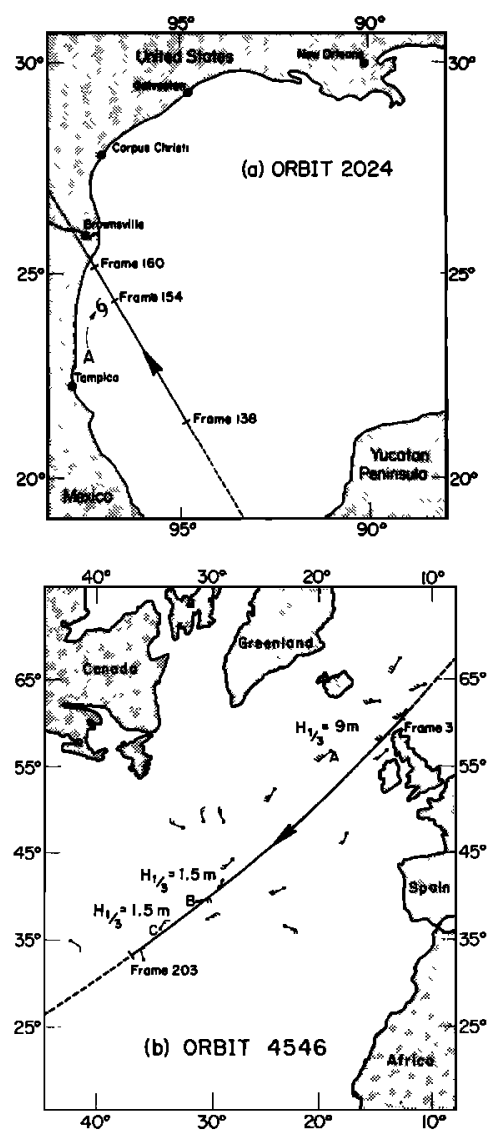

Fig. 6. Surface maps of subsatellite tracks. (a) Orbit 2024, Gulf of Mexico. Point A indicates the location of the hurricane eye (frame 154 corresponds to point of closest approach). (b) Orbit 4546, North Atlantic. Flags indicate surface winds. Points A, B, and C indicate location of ship weather reports.

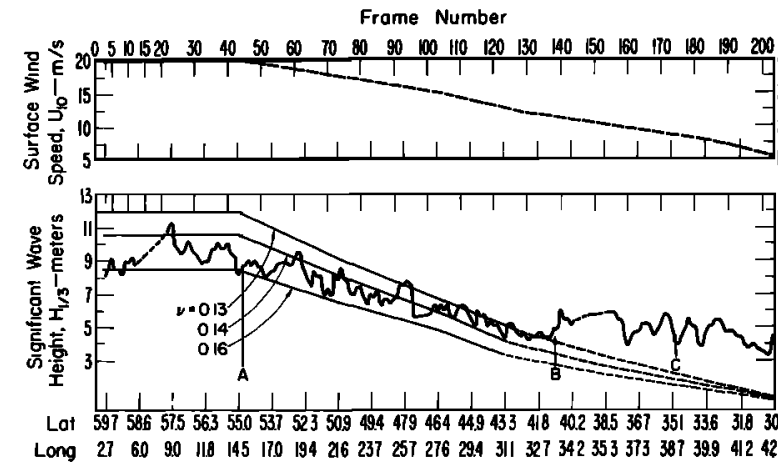

Fig. 7. Plot of significant wave height and surface winds along subsatellite path for orbit 4546 in the North Atlantic, February 25, 1976. $H_{1 / 9}$ is corrected for instrument jitter. Light lines show parametric model fit to $H_{1 / 3}$. Points $A, B$, and $C$ correspond to the points of closest approach to the ship weather reports.

where $U_{10}$ is the wind speed referred to a height of $10 \mathrm{~m}$. The surface winds taken at a 10-m height are assumed to be equal to the winds reported in the surface charts. Hasselmann's parametric model defines the wave height in terms of two parameters, $\nu$ and $U_{10}$; however, his model considers only wind-driven waves. When wind wave heights are small, swell wave heights may be dominant. Ship reports were used in conjunction with wind wave heights based on the parametric model to determine that the swell probably did not contribute significantly to the wave height for the ground track considered here.

The estimated values of the surface winds along the satellite track are given in Figure 7. The corresponding wave heights for the wind-driven sea are given using (13) for three values of peak frequency, $\nu=0.13,0.14$, and 0.16 . The fully developed case implies a $20-\mathrm{m} / \mathrm{s}$ wind blowing over a long fetch for approximately $36-48$ hours. That is consistent with the weather maps off the northern coast of Scotland. In contrast, $\nu=0.14$ and $\nu=0.16$ represent lower wave heights and shorter duration winds. The dotted lines in Figure 7 refer to the portion of the analysis when the wind waves are small and the $H_{1 / 3}$ measurements deviate from the model values. On the basis of the available surface truth and the results of Figure 7, the wave height is overestimated when $H_{1 / \mathrm{s}} \approx 4 \mathrm{~m}$; this discrepancy is thought to be caused by bias error which is known to be more sensitive to the lower wave heights. Other analysis methods and/or a slight adjustment of $\sigma_{\tau}$ could decrease this discrepancy.

The statistical significance of the $H_{1 / 8}$ estimates, analyzed in terms of the regression slope variance, is discussed in Appendix C. When $H_{1 / \mathrm{s}}>4 \mathrm{~m}$, the estimates of $H_{1 / 3}$ are within $\pm 1 \mathrm{~m}$ for $75 \%$ of the data. For this analysis, three frames were averaged for each data point which corresponds to spatial averaging over $70 \mathrm{~km}$.

\section{Conclusions}

A method of analyzing the Geos 3 radar altimeter pulses has been developed which gives estimates of $H_{1 / 9}$ with sufficient accuracy for global measurements, provided $H_{1 / 3} \approx 4 \mathrm{~m}$. However, this method requires that the altimeter data be properly edited to ensure this accuracy.

The small scatter in $H_{1 / s}$, standard deviation of $0.6 \mathrm{~m}$ or less, is in reasonable agreement with the statistical analysis, $\pm 1 \mathrm{~m}$ of the true values $75 \%$ of the time.

The bias errors in significant wave height are more sensitive to the analysis method including transmitter pulse width for 
the lower wave heights. The sensitivity to these errors should be improved with narrower pulse altimeters such as the one scheduled for launch on Seasat-A in May 1978. Indeed, the method employed here may be useful in developing techniques for analyzing altimeter pulses from the Seasat-A satellite scheduled for launch in May 1978.

\section{Appendix A. Statistical Analysis of Individual Pulses}

The purpose of this work has been to infer significant wave height from average pulse shape. However, additional information related to sea state and the validity of the theory is available from the statistical fluctuations of the individual pulses. Furthermore, these fluctuations can indicate when the altimeter is operating properly. The statistical analysis is separated into two categories: (1) the correlation analysis between individual pulses and gates and (2) the moments of the amplitude statistics of the individual gates.

The interpulse and intergate correlation analyses are useful in assessing the validity of theoretical assumptions and in checking on the spacing of the 16 sampling gates, respectively. The interpulse correlation analysis is useful, since one would expect that the amplitudes of the square law detected return pulses are uncorrelated from pulse to pulse. Normally, many specular points in the illuminated footprints contribute to the backscattering, causing uniform distributed phase between many instantaneous pulses [Berger, 1972]. However, an exception to this could occur in the gate, which measures the backscattered energy when the pulse first intersects the top of
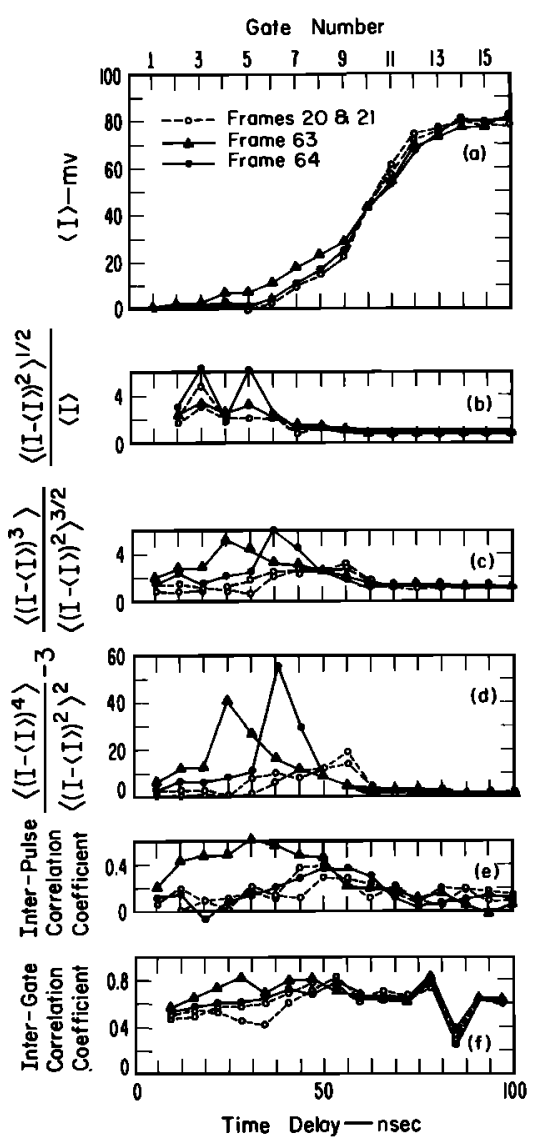

Fig. Al. Typical example for moments of statistical amplitude distribution, and intergate and interpulse correlation coefficients for four individual frames. Upper plot is average pulse shape, first-order moment; lower plots correspond to second-, third-, and fourth-order moments and correlation coefficients. the sea surface, i.e., when it has encountered only a few specular points. Theoretically, one would expect a detectable correlation in this one gate only for a sea with very long dominant waves, where the distance between specular points is large. But with the Geos 3 altimeter such a correlation may not be detectable, because even after correcting for jitter of the position of the gates, the 1.56-ns quantization level results in an indeterminacy of the illuminated area of $1.18 \mathrm{~km}^{2}$ from pulse to pulse. Even for large swell the pulse most likely illuminates at least 5-10 specular points, which assuming uniform distributed phase is usually sufficient to approach Gaussian statistics.

The intergate correlation analysis is one method of estimating the relative location of the individual gates. Indeed, an abnormally low intergate correlation coefficient would imply a larger than normal spacing between adjacent gates. The nominal altimeter design specifications are gates $12.5 \mathrm{~ns}$ wide, spaced 6.25 ns apart.

The correlation, for example, between gates 1 and 2 is calculated by using

$$
R_{12}=\frac{\sum_{j=1}^{320} y_{1 j} y_{2 j}}{\left\{\sum_{j=1}^{320} y_{1 j}^{2} \sum_{j=1}^{320} y_{2 j}^{2}\right\}^{1 / 2}}
$$

where

$$
\begin{array}{cc}
y_{1 j}=I_{1 j}-\mu & y_{2 j}=I_{2 j}-v \\
\mu=\frac{1}{320} \sum_{j=1}^{320} I_{1 j} & v=\frac{1}{320} \sum_{j=1}^{320} I_{2 j}
\end{array}
$$

$I_{1}, I_{2 j}$ are the instantaneous energies in gates 1 and 2, respectively. A similar procedure was used to calculate other intergate and the interpulse correlation coefficients.

Figure A1 illustrates typical variations in the correlation coefficients and statistical moments for four different sets of data. Two sets, designated as frames 20 and 21 , were selected for low seas, $H_{1 / 9} \approx 4 \mathrm{~m}$, whereas the remaining two sets of data were selected at times of higher seas, $H_{1 / 3} \cong 8 \mathrm{~m}$. Frame 64 is typical, whereas frame 63 is associated with a large negative spike in satellite altitude. These spikes have previously been considered to bias the estimates of $H_{1 / \mathrm{s}}$. Plot a shows the average pulse shape, first moment, for the four data sets, whereas plot $\mathrm{e}$ illustrates the variation in the interpulse correlation as a function of delay time or gate number. The correlation coefficients for the noise gates $1-6$ and the plateau gates 13-16 are smaller than those for the transitionary gates $7-12$, which have typical values of $0.2-0.3$. However, an exception occurs at times of large negative spikes in satellite altitude as illustrated by frame 63 , which displays coefficients 0.4-0.6 in the noise gates. Therefore the interpulse correlation is considered a useful indicator of negative spikes in altitude. Plot $f$ illustrates the variation in intergate correlation. Again the larger correlation for frame 63 is evident although not as

TABLE A1. Statistical Moments for Exponential Distribution

\begin{tabular}{lcc}
\hline & \multicolumn{2}{c}{ Distribution } \\
\cline { 2 - 3 } & $P(I)$ & $(1 / a) e^{-I / a}$ \\
\hline First moment & $\langle I\rangle$ & $a$ \\
Second moment & $\left\langle\left(I-\langle I)^{2}\right\rangle^{1 / 2} /\langle I\rangle\right.$ & 1 \\
Third moment & $\left\langle(I-\langle I\rangle)^{3}\right\rangle /\left\langle(I-\langle I\rangle)^{2}\right\rangle^{\mathrm{a} / 2}$ & 2 \\
Fourth moment & {$\left[\left(\left(I-\langle I)^{4}\right\rangle /(I I-\langle I\rangle)^{2}\right\rangle^{2}\right]-3$} & 6 \\
\hline
\end{tabular}




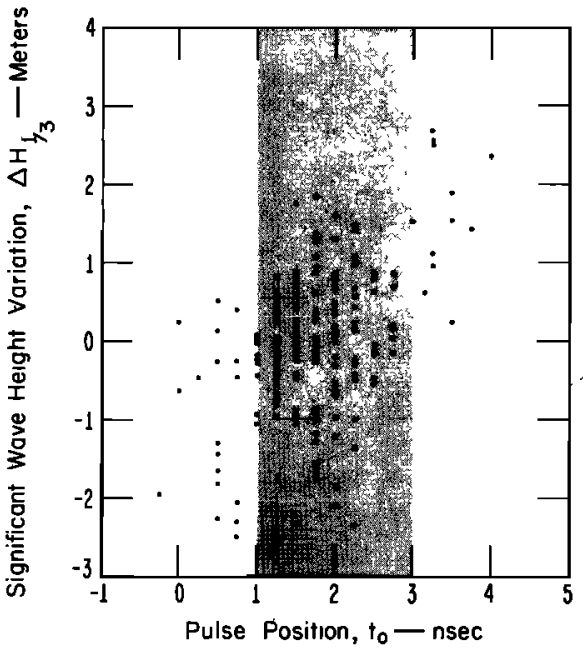

Fig. B1. Mass plot showing relationship between changes in wave height and pulse position.

pronounced. The consistent unexpected decrease in correlation between gates 13 and 14 and the increase in correlation between gates 12 and 13 are interpreted as a mislocation of gate 13 such that it is nearly in the same location as gate 12 . The remaining lower plots illustrate the variations in the individual gate amplitude distributions. The expected values based on Gaussian statistics and the square law detector result in an exponential distribution for the individual gates. The expected values for the first four moments are given in Table A1. The second moment is a first indicator of how close the amplitude statistics can be approximated by exponential distribution, since the mean equals the standard deviation for this distribution. The large values of the second moment for the noise gates should be ignored, since $\langle I\rangle$ may be $\cong 0$ for these gates. The third moment, skewness, and the fourth moment, kurtosis, give more detailed information about the amplitude statistics.
These parameters are higher for the high sea state conditions, which suggests that they may be a measure of sea state, changes in altitude, or perhaps some other related effect.

\section{A ppendix B. Observational Biases in $H_{1 / \mathrm{g}}$ Estimates}

Some scatter in the values of $H_{1 / \mathrm{s}}$ is expected because the results are only estimates of the true values: The statistical significance of the estimates can be calculated (see Appendix C). The purpose of this section is to show that other systematic factors can bias these $H_{1 / 3}$ estimates.

The results for orbits 2024 and 4546 were carefully analyzed in an attempt to determine if a relationship could be found between the large positive and/or negative excursion from the trends in $H_{1 / 3}$ and other altimeter-related parameters. This analysis showed a definite relationship between both positive and negative excursions in $H_{1 / 3}$, hereafter designated $\Delta H_{1 / 3}$, and the position of the average received pulse, $t_{0}$. Figure B1 illustrates the correlation between $\Delta H_{1 / 8}$ and $t_{0}$ for orbit 4546; indeed, typical pulse positions are $2 \mathrm{~ns}$, larger delays being associated with positive excursions of $H_{1 / 3}$ and smaller delays with negative excursions. The correlation between $\Delta H_{1 / \mathbf{a}}$ and $t_{0}$ as illustrated in Figure $B l$ is 0.78 provided that only values associated with $t_{0}>3 \mathrm{~ns}$ and $t_{0}<1 \mathrm{~ns}$ are considered in the analysis. Therefore the largest and smallest values of $t_{0}$ bias the estimates of $H_{1 / \mathrm{g}}$. The data associated with $t_{0}>3 \mathrm{~ns}$ and $t_{0}<1$ ns were deleted from the data analyzed here.

\section{Appendix C. Statistical Significance of $H_{1 / 9}$ Estimates}

The random variations in the estimates of $H_{1 / 9}$ are important in determining the measurement accuracy of the altimeter. The estimates of $H_{1 / 9}$ are obtained by a least square fit of an error function to five data points defining the pulse shape near its midrange. The argument of the error function is related to $H_{1 / 3}$ by $(9)$ and (10). The purpose of this section is to determine the statistical significance of the estimates. However, since a direct method using the error function is mathemati-

TABLE C1. Statistical Analysis of Regression Line for $H_{1 / 3}$, Orbit 2024

\begin{tabular}{|c|c|c|c|c|c|c|c|}
\hline \multirow[b]{2}{*}{ Frame } & \multirow{2}{*}{$\begin{array}{c}\text { Slope Estimate } \\
b, \mathrm{mV} / \mathrm{ns}\end{array}$} & \multirow{2}{*}{$\begin{array}{c}\text { Standard } \\
\text { Deviation } \\
\text { of Slope } \\
\text { Estimate } S(b), \\
\mathrm{mV} / \mathrm{ns}\end{array}$} & \multirow[b]{2}{*}{ Constant $d$} & \multirow{2}{*}{$\begin{array}{c}\text { Standard } \\
\text { Deviation } \\
\text { of } H_{1 / 3} \\
\text { Estimates } \\
S\left(H_{1 / 3}\right), \mathrm{m}\end{array}$} & \multicolumn{2}{|c|}{ 75\% Confidence Limits } & \multirow[b]{2}{*}{$H_{1 / \mathrm{s}}, \mathrm{m}$} \\
\hline & & & & & Lower & Upper & \\
\hline 138 & 3.26 & 0.34 & 1369 & 2.18 & -2.3 & 6.72 & 2.21 \\
\hline 139 & 3.37 & 0.24 & 1436 & 1.40 & -0.59 & 5.21 & 2.31 \\
\hline 140 & 3.02 & 0.30 & 1402 & 1.52 & 0.48 & 6.76 & 3.62 \\
\hline 141 & 3.05 & 0.23 & 1380 & 1.10 & 1.38 & 5.94 & 3.66 \\
\hline 142 & 3.33 & 0.25 & 1425 & 1.39 & -0.38 & 5.37 & 2.49 \\
\hline 143 & 3.02 & 0.25 & 1425 & 1.18 & 1.52 & 6.40 & 3.96 \\
\hline 144 & 3.45 & 0.26 & 1391 & 3.05 & -5.27 & 7.35 & 1.04 \\
\hline 145 & 3.23 & 0.21 & 1414 & 1.03 & 0.95 & 5.21 & 3.08 \\
\hline 146 & 2.35 & 0.20 & 1380 & 1.08 & 4.82 & 9.28 & 7.05 \\
\hline 147 & 3.23 & 0.21 & 1391 & 1.07 & 0.71 & 5.13 & 2.92 \\
\hline 148 & 2.90 & 0.20 & 1369 & 0.90 & 2.61 & 6.33 & 4.47 \\
\hline 149 & 3.14 & 0.23 & 1391 & 1.13 & 0.96 & 5.64 & 3.30 \\
\hline 150 & 2.87 & 0.22 & 1380 & 1.02 & 2.42 & 6.64 & 4.53 \\
\hline 151 & 2.87 & 0.24 & 1380 & 1.14 & 2.08 & 6.80 & 4.44 \\
\hline 152 & 2.96 & 0.20 & 1369 & 0.94 & 2.08 & 5.97 & 4.03 \\
\hline 153 & 2.46 & 0.15 & 1369 & 0.76 & 4.98 & 8.12 & 6.55 \\
\hline 154 & 2.76 & 0.17 & 1406 & 0.79 & 3.55 & 6.81 & 5.18 \\
\hline 155 & 2.32 & 0.15 & 1376 & 0.83 & 5.49 & 8.93 & 7.21 \\
\hline 156 & 2.25 & 0.21 & 1421 & 1.21 & 5.28 & 10.3 & 7.78 \\
\hline 157 & 2.19 & 0.15 & 1440 & 0.94 & 5.94 & 9.82 & 7.78 \\
\hline 158 & 2.49 & 0.15 & 1428 & 0.76 & 5.02 & 8.16 & 6.59 \\
\hline 159 & 2.50 & 0.19 & 1325 & 0.96 & 4.07 & 8.04 & 6.06 \\
\hline
\end{tabular}


cally complicated, for simplicity a linear regression analysis is applied and shown to be a good approximation.

If two variables can be correlated linearly, the regression coefficient is the slope of the line used to correlate the variables. We are interested in the estimated deviations of this slope which can easily be related to $H_{1 / 3}$. If higher-order correlation is required (i.e., terms of order higher than linear can be obtained by using a series expansion of the error furction), then a least square error analysis would give regression coefficients for each of the higher-order regression coefficients, a socalled curvilinear regression analysis. The curvilinear method is useful, since it permits the stepwise removal of each successive lower degree of the error function series expansion such that the calculation can be terminated at any level when the regression is adequate. Therefore in practice one need show only that the first few higher-order terms are small in comparison with the first-order (linear) term to establish that the linear regression is a valid method of determining the confidence bounds.

The curvilinear regression analysis was applied to selected frames of data from orbit 2024 by using a method described by Volk [1969]. The results showed that second-degree and thirddegree terms may be removed with negligible error. Therefore a linear regression analysis is deemed sufficient to determine the statistical significance of the $H_{1 / 8}$ estimates. The error bars are obtained by applying a standard regression analysis, in terms of the least squares line $I=a+b t$, over the range of the five data points, where $b$ is the slope estimate or regression coefficient. The results of this analysis give a correlation coefficient between 0.98 and 0.99 , which is in agreement with the curvilinear regression result that only linear regression is required. The regression analysis gives the standard deviation of this slope estimate, $S(b)$. However, a transformation is required to convert to the standard deviation of $H_{1 / 3}, S\left(H_{1 / 8}\right)$. Using (10) in a slightly different form we have

$$
H_{1 / 8}=0.6\left[d / b^{2}-\left(\sigma_{\tau}{ }^{\prime}\right)^{2}\right]^{1 / 2}
$$

where $d=\left[\left(I_{\infty}-I_{-\infty}\right)^{2}\right] / 2 \pi$ and $\sigma_{\tau}{ }^{\prime}=7.8 \mathrm{~ns}$. Then, using $S\left(H_{1 / 3}\right)=\left(d H_{1 / 3} / d b\right) S(b)$, we obtain

$$
S\left(H_{1 / 3}\right)=\frac{0.36 d}{b^{3} H_{1 / 3}} S(b)
$$

Table $\mathrm{Cl}$ shows the relevant statistical parameters for each frame of orbit 2024. The confidence limits are given as $H_{1 / 9} \pm$ $t S\left(H_{1 / 8}\right)$, which for a $75 \%$ chance of being within the range $H_{1 / 3}$ $\pm t S\left(H_{1 / 8}\right)$ gives $t=2.069$. The results in Table $\mathrm{Cl}$ illustrate the high percent of change in the confidence limits for the lower sea states. Furthermore, the lowest values of $H_{1 / 3}$, such as the value for frame 140 , which are normally deleted from the analysis using the editing criteria discussed above, give the most unreliable estimates of $H_{1 / 3}$. The estimates of $H_{1 / 9}$ can be considered to be statistically significant within about $\pm 2 \mathrm{~m}$ for $75 \%$ of the data when $H_{1 / \mathrm{s}} \sim 4 \mathrm{~m}$ for spatial averaging over 23 $\mathrm{km}$.

The $H_{1 / 3}$ estimates are statistically more significant for orbit 4546 , since a running average of three points (frames) was used. Indeed, $S\left(H_{1 / 3}\right)$ will be smaller by a factor $(3)^{1 / 2}$, i.e., $S_{\mathrm{s}}\left(H_{1 / 8}\right)=0.577 S_{1}\left(H_{1 / 8}\right)$, or in other words the confidence interval will be reduced by a factor of about 2 from the values of Table Cl. When $H_{1 / 3} \approx 4 \mathrm{~m}$, the estimates of $H_{1 / 8}$ can be considered to fall within $\pm 1 \mathrm{~m}$ for $75 \%$ of the data for spatial averaging over $70 \mathrm{~km}$.

Acknowledgments. The authors wish to thank K. Hasselmann and Duncan Ross for their encouragement and guidance during this work. The computer programing help from Bruce Gritton, Allan Yanaway, and Ruth Alpers is kindly acknowledged. This work was partially supported by NATO research grant SRG/AI.10., NOAA contract 03 022-35163, and NASA contract 855-33-05-09-53.

\section{REFERENCES}

Barrick, D. E., Rough surface scattering based on the specular point theory, IEEE Trans. Antennas Propagat., 16(4), 449-454, 1968.

Barrick, D. E., Remote Sensing of the Troposphere, chap. 12, edited by V. E. Derr, U.S. Government Printing Office, Washington, D. C., 1972.

Berger, T., Satellite altimetry using ocean backscatter, IEEE Trans. Antennas Propagat., 20(3), 295-309, 1972.

Brown, G.S., The average impulse response of a rough surface and its applications, IEEE Trans. Antennas Propagat., 25(1), 67-74, 1977.

Cardone, V. J., W. J. Pierson, and E. G. Ward, Hindcasting the directional spectra of hurricane-generated waves, J. Petrol. Technol., 385-394, 1976.

Hasselmann, K., D. B. Ross, P. Muller, and W. Sell, A parametric wave prediction model, J. Phys. Oceanogr., 6(2), 200-228, 1976.

Hofmeister, E. L., B. N. Keeney, T. W. Godbey, and R. J. Berg, Data Users Handbook and Design Error Analysis, Aircraft Equipment Division, General Electric Company, Utica, N. Y., 1976.

Kinsman, B., Wind Waves: Their Generation and Propagation on the Ocean Surface, Prentice-Hall, Englewood Cliffs, N. J., 1965.

McGoogan, J. T., Satellite altimetry applications, IEEE Trans. Microwave Theory Tech., 23(12), 970-978, 1975.

Moore, R. K., and C. S. Williams, Jr., Radar terrain at near vertical incidence, Proc. IRE, 45, 228-238, 1957.

Pierson, W. J., Comments on a parametric wave prediction model, $J$. Phys. Oceanogr., 7(1), 127-137, 1977.

Shapiro, A., E. A. Uliana, and B. S. Yaplee, Radar pulse shape versus ocean wave height, edited by J. R. Apel, Tech. Rep. ERL 228$A O M L$ 7, p. 11-1, Nat. Oceanic and Atmos. Admin., Washington, D. C., 1972.

Volk, W., Applied Statistics for Engineers, 2nd ed., pp. 323-328, McGraw-Hill, New York, 1969.

(Received November 8, 1977; revised March 30, 1978; accepted April 7, 1978.) 